\title{
Mapping Anti-phase Domains by Polarity Sensitive Orientation Imaging Using Electron Backscatter Diffraction
}

\author{
G. Naresh-Kumar ${ }^{1}$, S. Vespucci ${ }^{1}$, A. Vilalta-Clemente ${ }^{2}$, H. Jussila ${ }^{3}$, A. Winkelmann ${ }^{4}$, G. Nolze ${ }^{5}$, S. \\ Nagarajan $^{3}$, A. J. Wilkinson ${ }^{2}$ and C. Trager-Cowan ${ }^{1}$ \\ ${ }^{1 .}$ Department of Physics, SUPA, University of Strathclyde, Glasgow, UK \\ 2. Department of Materials, University of Oxford, Parks Road, Oxford, UK \\ ${ }^{3 .}$ Department of Micro and Nanosciences, Aalto University, Aalto, Finland \\ 4. Bruker Nano GmbH, Am Studio 2D, Berlin, Germany \\ 5. BAM, Unter den Eichen 87, Berlin, Germany
}

Various material properties such as piezoelectricity, spontaneous polarisation, and plasticity are directly dependent on the crystal structure, and any form of deviation from their perfect crystal lattice could significantly alter their fundamental behaviour. Producing defect free materials is a challenging task especially in the case of heteroepitaxial thin film growth. Irrespective of the substrates, the growth plane, or the growth conditions employed, extended defects such as dislocations, stacking faults and grain boundaries are generally observed in the as-grown layers. In addition to these commonly observed extended defects; inversion domains (IDs), antiphase domains (APDs) and antiphase boundaries (APBs) have also been identified in several materials; examples include, layered perovskite structured materials, semiconductors, metallic superlattices and shape memory alloys. Often extended defects are electrically active and are problematic for electronic and optoelectronic devices. This is why structural characterisation techniques which are simultaneously rapid to use, non-destructive and structurally definitive on the nanoscale become a prerequisite.

In our presentation, we will demonstrate the application of using electron backscatter diffraction (EBSD) in a field emission scanning electron microscope (FE-SEM) to image and quantify APDs in a single crystalline GaP thin film grown on $\mathrm{Si}$ substrates with different offcuts. We were able to image and quantify APDs by relating the asymmetrical intensity distributions observed in the EBSD patterns acquired experimentally and comparing the same with the dynamical electron diffraction simulations [1, 2]. Additionally misorientation maps were measured using automated cross-correlation based approaches [3]. Samples grown on substrates with a $4^{\circ}$ offcut from the [110] do not show any APDs, whereas samples grown on the exactly oriented substrates contain APDs. The procedures described in our work can be adopted for characterising a wide range of other material systems possessing noncentrosymmetric point groups.

\section{References:}

[1] Winkelmann, A. and Nolze, G., Applied Physics Letters. 106 (2015), p. 072101.

[2] Nolze, G. Grosse, C. and Winkelmann, A. Journal of Applied Crystallography. 48 (2015), p. 1405.

[3] Wilkinson, A. J., Meaden, G. and Dingley, D. J. Ultramicroscopy. 106 (2006), p. 307. 


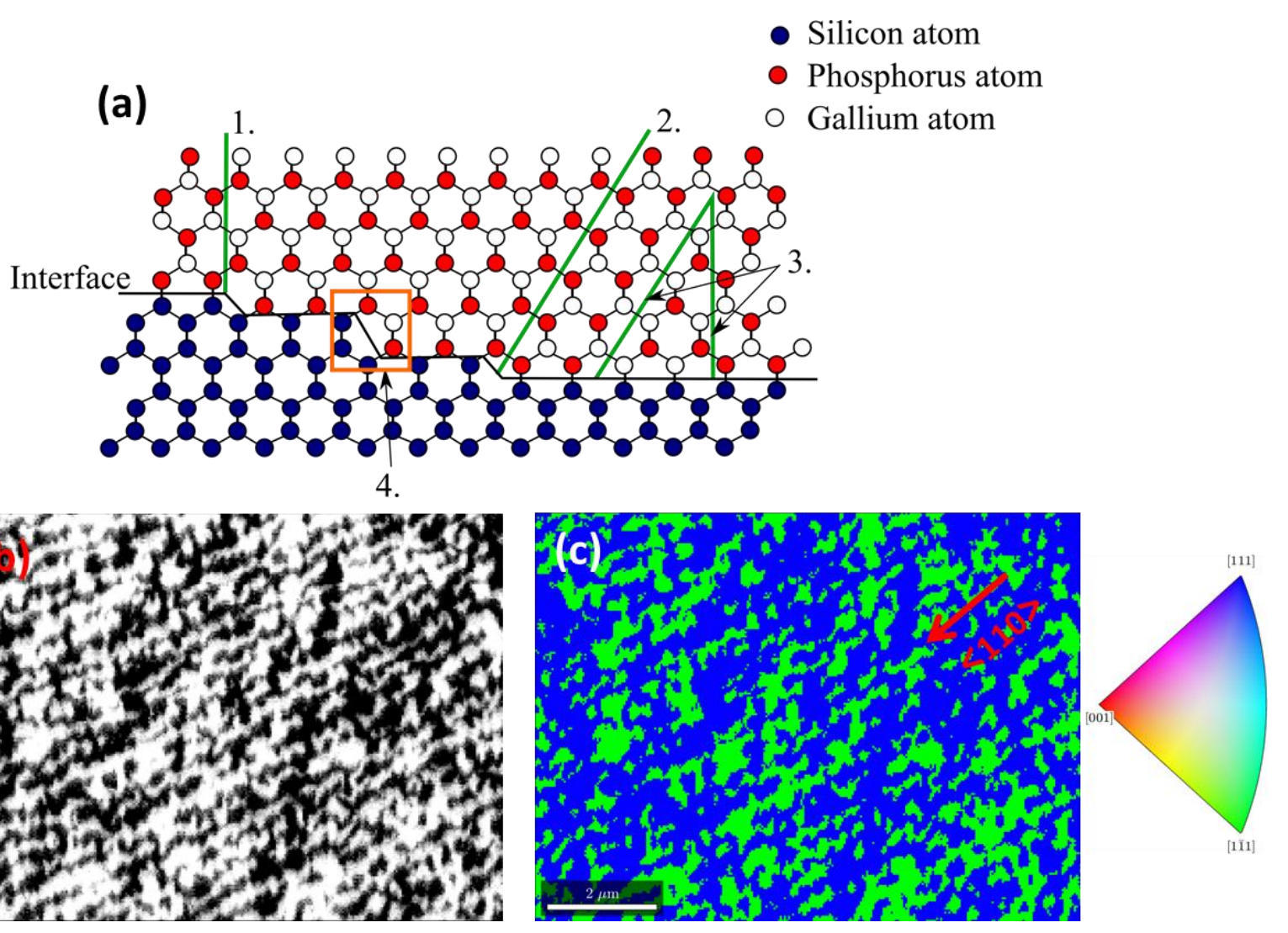

Figure 1. Antiphase domains in $\mathrm{GaP}$ on Si substrates. (a) Ball and stick model showing the formation and annihilation of antiphase boundaries (APBs) in GaP grown on a Si substrate,

(1.) APBs along (110) due to sub-lattice occupation disorder, (2.) APBs along the $\{111\}_{\text {Si }}$ due to monoatomic steps, (3.) annihilation of APBs along (111) and (110) and (4.) annihilation of APBs due to diatomic steps, (b) Region of interest asymmetry imaging from the $\{111\}$ bands produced from the recorded EBSD patterns. The bright and dark regions are the regions of two different domains, (c) the EBSD inverse pole figure (IPF) map for the sample reference direction [111] revealing the APDs. Regions with APDs are coloured green and blue according to the IPF colour key, which indicates a $90^{\circ}$ rotation around [001] from $\langle 111\rangle$ to the non-equivalent $\langle 1 \overline{1} 1\rangle$. The step structures (of the order of $100 \mathrm{~nm}$ ) can also be seen along $\langle 110\rangle$. 